\title{
Vor 60 Jahren: der Haager Europa-Kongress
}

\author{
Wilfried Loth*
}

Vom 7. bis zum 10. Mai 1948 trafen sich 722 repräsentative Persönlichkeiten aus 28 europäischen Ländern in Den Haag, um über Wege zu einer Einigung Europas zu diskutieren. Sechs ehemalige Premierminister europäischer Länder nahmen an der Veranstaltung teil, 14 aktive und 45 ehemalige Minister, westdeutsche Ministerpräsidenten, führende Abgeordnete, Wirtschaftsführer, Gewerkschafter, Kirchenführer, zahlreiche Professoren, einige Intellektuelle und Künstler. Winston Churchill hielt die Eröffnungsansprache; etwa 40.000 Menschen kamen zu einer öffentlichen Kundgebung am dritten Verhandlungstag. Der Kongress führte zur Konstituierung der Europäischen Bewegung und mittelbar auch zur Gründung des Europarates. ${ }^{1}$

60 Jahre später, in einer Zeit unsicheren Wartens auf die Ratifizierung des Vertrags von Lissabon, erscheint der Haager Kongress entweder als Sinnbild einer längst vergangenen Zeit, in der die Europa-Idee noch Begeisterung hervorzurufen vermochte und politische Kraft entfalten konnte oder als Marginalie in einer Geschichte der europäischen Integration, die im Wesentlichen von nationalen Regierungen geschrieben wurde und ihren Interessenausgleich widerspiegelt. Tatsächlich war er weder das Eine noch das Andere. Sein Zustandekommen und sein Verlauf zeigen vielmehr, dass nationale Regierungen stets der Vernetzung gesellschaftlicher Kräfte auf europäischer Ebene bedürfen, wenn sie europapolitisch erfolgreich sein wollen.

\section{Winston Churchill und Duncan Sandys}

Die Konstituierung einer länderübergreifenden Europäischen Bewegung, die sogleich Einfluss auf die Politik einiger europäischer Regierungen nahm, war das Ergebnis von Sammlungsbemühungen, die von Churchill ausgingen. Der ehemalige britische Kriegspremier, seit den Unterhauswahlen vom Juli 1945 eher lustloser Führer der konservativen Opposition, betrachtete es als seine Aufgabe, die Einigung der westlichen Länder auf dem europäischen Kontinent zu fördern - als Bollwerk gegen die Gefahr einer sowjetischen Expansion ebenso wie als Voraussetzung für wirtschaftliche Gesundung und Stabilisierung der Demokratie. In einer aufsehenerregenden Rede vor Züricher Studenten im Oktober 1946 forderte er „eine Art Vereinigte Staaten von Europa zu schaffen“, beruhend auf einer „Partnerschaft von Frankreich und Deutschland“. Großbritannien sah er dabei eher unter den „Förderern des neuen Europa“ als unter seinen Mitgliedern; freilich sollte es eine höchst aktive Rolle bei seiner Konstituierung spielen. ${ }^{2}$

Um die Mobilisierung der öffentlichen Meinung voranzutreiben, beauftragte Churchill seinen Schwiegersohn und engen politischen Mitstreiter Duncan Sandys mit der Organisation einer überparteilichen Gruppe repräsentativer Persönlichkeiten, die den europäischen

* Prof. Dr. Wilfried Loth, Lehrstuhl für Neuere und Neueste Geschichte, Historisches Institut der Universität Duisburg-Essen.

1 Zu Vorgeschichte und Verlauf des Haager Kongresses vgl. Frank Niess: Die europäische Idee aus dem Geist des Widerstands, Frankfurt/Main 2001, S. 158-173 und 181-220; zum Kontext Wilfried Loth: Der Weg nach Europa. Geschichte der europäischen Integration 1939-1957, Göttingen 1990, 3. Aufl. 1996.

2 Rede vom 19.9.1946, in: Walter Lipgens/Wilfried Loth (Hrsg.): Documents on the History of European Integration, Vol. III: The Struggle for European Union by Political Parties and Pressure Groups in Western European Countries, 1945-1950, Berlin/New York 1988, S. 662-666. 
Einigungsgedanken in Großbritannien fördern sollten. Sandys Bemühungen trugen bald Früchte: Am 16. Januar 1947 konnte er ein provisorisches ,British United Europe Committee' präsentieren, dem neben konservativen Abgeordneten (unter anderen Robert Boothby) auch Labour-Politiker und Gewerkschaftsvertreter (Gordon Land, George Gibson, Victor Gollancz), Vertreter der liberalen Partei, der Kirchen und der Wissenschaft (darunter Bertrand Russell) angehörten; die britischen Föderalisten waren unter anderen durch Frances L. Josephy vertreten. Allerdings sprach sich das Exekutivkomitee der Labour Party gegen das Unternehmen aus, weil es weder Churchills Idee einer westlichen Blockbildung fördern noch dem Oppositionsführer eine Plattform für innenpolitische Erfolge bieten wollte. Infolgedessen entwickelten sich die Aktivitäten der Gruppe, die sich dann am 14. Mai 1947 definitiv als ,United Europe Movement ${ }^{\star}$ (UEM) konstituierte, vorwiegend im konservativen und liberalen Milieu. ${ }^{3}$

Parallel zum UEM organisierten der ehemalige belgische Ministerpräsident Paul van Zeeland und Joseph Retinger, langjähriger Mitarbeiter des polnischen Exilpremiers Wladyslaw Sikorski, in Belgien, Luxemburg, Großbritannien und Frankreich eine ,Independent League of European Co-operation“ (ILEC), die an die europäischen Zollunions-Komitees der 1920er und 1930er Jahre anknüpfte. Am 7. März 1947 konnten sie die Konstituierung eines provisorischen Zentralkomitees auf internationaler Ebene bekannt geben. Die Gruppe sammelte einflussreiche Wirtschaftswissenschaftler, Bankiers und Manager, denen die Behinderung des Wiederaufbaus durch nationale Wirtschaftsgrenzen in Europa Sorgen machte. Sie teilten zwar keineswegs alle Churchills Furcht vor einer sowjetischen Expansion. Da sie aber auf einen raschen Beginn wirtschaftlicher Integration ohne Rücksicht auf sowjetische Vorbehalte drängten und ebenso wenig auf eine bestimmte Integrationsmethode festgelegt waren wie der britische Oppositionsführer, waren sie für eine Zusammenarbeit mit der Sandys-Gruppe geradezu prädestiniert. Manche Politiker, so der ehemalige Direktor des Internationalen Arbeitsamtes Harold Butler und der spätere britische Premierminister Harold Macmillan, wurden in beiden Organisationen zugleich aktiv. ${ }^{4}$

Im Gefolge der Churchill-Rede wurde schließlich auch Richard Graf CoudenhoveKalergi, der Begründer der Paneuropa-Bewegung, wieder in der europäischen Politik aktiv. Zunächst schlug er Churchill eine Wiederbelebung der Paneuropa-Union ,,unter unserer gemeinsamen Führung" vor. Nachdem dieser aber zurückhaltend reagiert hatte, organisierte Coudenhove im November 1946 eine Umfrage unter den Parlamentariern des westlichen Europa. Über 4.000 Abgeordnete wurden gebeten, sich - zustimmend oder ablehnend - zu der Frage zu äußern, ob sie ,eine europäische Föderation im Rahmen der Vereinten Nationen" befürworteten. Damit sollte die Einigungsbereitschaft in den Ländern des westlichen Europa demonstriert und Druck auf die Regierungen ausgeübt werden, endlich mit Initiativen zur Schaffung eines westlichen Europas zu beginnen. Die zustimmenden Abgeordneten wurden aufgefordert, in den Parlamenten überparteiliche Komitees zu bilden, die sich dann im Juni 1947 zu einem Europäischen Kongress in Genf treffen sollten. ${ }^{5}$

Die Aktion zeigte freilich, dass die Idee eines Zusammenschlusses, der das östliche Europa von vorneherein ausschloss und so die begonnene Spaltung Europas vertiefte, im Winter 1946/47 noch nicht sonderlich populär war. Nur wenige Abgeordnete waren bereit, sich mit einem solchen Konzept zu identifizieren. Bis Ende April 1947 gingen 660 Antworten

3 Vgl. Walter Lipgens: Die Anfänge der europäischen Einigungspolitik 1945-1950. Erster Teil: 1945-1947, Stuttgart 1977, S. 313-331; Niess: Europäische Idee, 2001, S. 131-144.

4 Lipgens: Anfänge, 1977, S. 331-339.

5 Ebenda, S. 438-444. 
bei Coudenhove ein; davon waren 646 positiv - kaum mehr als ein Achtel der erbetenen Antworten. Die ambitiösen Kongresspläne mussten folglich vorerst vertagt werden. Ähnlich erfolglos verliefen die Bemühungen René Courtins, Mit-Herausgeber von ,Le Monde', in Frankreich ein Parallelkomitee zu dem britischen UEM zustande zu bringen. Die französischen Europa-Anhänger scheuten zumeist das Risiko, mit Churchills Westblock-Konzeption in Verbindung gebracht zu werden. ${ }^{6}$ Die Stimmen, die eine Einigung auch ohne sowjetische Zustimmung befürworteten, wurden zwar allmählich zahlreicher, doch überwog die negative Reaktion auf Churchills Vorstoß insgesamt bei Weitem. ${ }^{7}$

Erst nach der sowjetischen Absage an den Marshall-Plan am 2. Juli 1947 wurde das anders. Die vielen Anhänger eines Europas der „Dritten Kraft“, das zwischen den USA und der Sowjetunion vermitteln sollte, rangen sich jetzt zu der Einsicht durch, dass die europäische Einigung realpolitisch nur noch im Westen beginnen konnte; und allgemein wuchs die Überzeugung, dass im Hinblick auf den europäischen Wiederaufbau und die Integration des westlichen Deutschlands nicht mehr viel Zeit zu verlieren war. Courtin konnte am 16. Juli 1947 die Gründung eines ,Conseil français pour l'Europe unie' bekannt geben, der sich als französisches Pendant zu Churchills UEM verstand. Führende Vertreter der französischen Sozialisten erklärten sich zur Mitarbeit bereit, so Robert Lacoste, Francis Leenhardt, André LeTrocquer und Ministerpräsident Paul Ramadier. Die Christdemokraten waren unter anderem durch Paul Coste-Floret, François de Menthon und Pierre-Henri Teitgen vertreten, die Linksliberalen durch Paul Bastid und René Mayer, die Unabhängigen Republikaner durch Paul Reynaud und René Mayer, die sozialliberale UDSR durch Edouard Bonnefous. Den Ehrenvorsitz übernahm Edouard Herriot, der langjährige Ministerpräsident der III. Republik. ${ }^{8}$

Coudenhoves Umfrage erhielt jetzt viel größere Resonanz. Nachdem er die Abgeordneten, die bislang noch nicht geantwortet hatten, im April 1947 noch einmal gemahnt hatte, erhöhte sich die Zahl der positiven Antworten bis Ende September auf 1.735. Damit sprachen sich insgesamt 43 Prozent der angeschriebenen Abgeordneten im Prinzip für eine ,europäische Föderation“" aus, darunter 64 Prozent der italienischen Abgeordneten, 53 Prozent der niederländischen Abgeordneten und je 50 Prozent der französischen und belgischen Abgeordneten. Von den britischen Abgeordneten reagierten allerdings nur 26 Prozent positiv, von den skandinavischen Abgeordneten sogar nur 12 Prozent. ${ }^{9}$ Nachdem sich in Frankreich, Belgien, Italien und Griechenland föderalistische Parlamentarier zu sammeln begonnen hatten, konnte Coudenhove-Kalergi vom 8. bis 10. September 1947 an seinem Wohnsitz in Gstaad zwar kein „Vorparlament“, aber immerhin eine Versammlung von 114 aktiven Abgeordneten aus zehn Ländern organisieren. Diese gründeten eine ,Europäische Parlamentarier-Union“ (EPU) und beschlossen, für die Einberufung einer Europäischen Verfassunggebenden Versammlung zu arbeiten. ${ }^{10}$

Für Duncan Sandys kam es nun darauf an, die Einigungsbewegung in den verschiedenen Ländern nicht nur zu stärken, sondern auch unter Kontrolle zu halten. Er war davon überzeugt, dass die Bewegung nur dann erfolgreich sein konnte, wenn sie sich zunächst auf eine funktionale Zusammenarbeit der Regierungen konzentrierte: Nur dann schien ihm eine britische Beteiligung erreichbar zu sein. Ohne Großbritannien, fürchtete er, würde es Frankreich

Ebenda, S. 570-571; Niess: Europäische Idee, 2001, S. 145-147.

Vgl. die Sammlung unterschiedlicher Reaktionen bei Lipgens: Anfänge, 1977, S. 339-343 und 435-438.

Ebenda, S. 570-573.

Ebenda, S. 441-444.

10 Ebenda, S. 548-561; Heribert Gisch: The European Parliamentary Union, in: Walter Lipgens/Wilfried Loth (Hrsg.): Documents on the History of European Integration, Vol. IV: Transnational Organizations of Political Parties and Pressure Groups in the Struggle for European Union, 1945-1950, Berlin/New York 1990, S. 112-185. 
nicht wagen, einem starken Westdeutschland in einer europäischen Gemeinschaft gegenüberzutreten. Folglich war für ihn, viel eindeutiger als für seinen Schwiegervater, eine britische Beteiligung an dem Einigungswerk unverzichtbar. ${ }^{11}$ Schon im Vorfeld der Konstituierung des ,Conseil français‘ lud er darum die übrigen Europa-Verbände zur Bildung eines „Verbindungskomitees“ der Europa-Bewegungen ein. Sie erfolgte am 20. Juli 1947 in Paris, im Rahmen eines Mittagessens auf den Champs Elysées. Neben dem UEM, dem französischen Rat, der ILEC und der EPU waren auch die militanten Föderalisten um Hendrik Brugmans und Alexandre Marc vertreten, die sich im Dezember 1946 zur ,Union Européenne des Fédéralistes‘ (UEF) zusammengeschlossen hatten. ${ }^{12}$

\section{Das Ringen um den Kongress}

Die Föderalisten waren davon überzeugt, dass die Zeit für eine föderale Neuorganisation der Völker Europas reif war. Ihnen schwebte daher die Einberufung von „Generalständen Europas“ vor, die sich gegen die nationalen Regierungen und Parlamente zur Verfassunggebenden Versammlung des Vereinten Europas entwickeln sollten. Basierend auf einer umfassenden Mobilisierungskampagne sollten dort die unterschiedlichen gesellschaftlichen Gruppen vertreten sein, „etwa Angestellte, Arbeiter, Landwirte, Vertreter der Mittelklasse, Verbraucherorganisationen, politische und parlamentarische Körperschaften, Jugendbewegungen“. Die ,spektakuläre Versammlung“" sollte nicht nur ,die öffentliche Meinung beeindrucken“, sondern auch „ständige Ausschüsse“" zur Bearbeitung der anstehenden rechtlichen, sozialen, wirtschaftlichen, kulturellen und weiteren Fragen installieren. Die Vorsitzenden dieser Ausschüsse sollten ,den Kern einer künftigen europäischen Regierung bilden. "13 Als Tagungsort für diese revolutionäre Manifestation wurde Versailles ins Auge gefasst.

Für Sandys waren diese Pläne eines korporatistischen Föderalismus gefährliche Hirngespinste, die die europäische Einigungsbewegung zu diskreditieren und die Chancen für eine Mitwirkung Großbritanniens an dem Einigungsprozess zunichte machen würden. Noch ehe die UEF-Führer mit der organisatorischen Umsetzung ihres Vorhabens beginnen konnten, vereinbarte er darum mit der Führung der ILEC Ende September 1947 die Vorbereitung eines ganz anders gearteten Kongresses: einer „Konferenz von 500 bis 800 prominenten Europäern“, die ,am ersten Wochenende nach Ostern 1948“ zusammentreten sollte, um die europäischen Regierungen zu drängen und zu ermutigen, mit ersten Schritten zur Einigung Europas zu beginnen. Der niederländische Senator und ehemalige Wirtschaftsminister Pieter Kerstens, der sich um die Konstituierung einer niederländischen Sektion des ILEC bemühte, sagte zu, die nötigen Gelder für die Finanzierung eines solchen Kongresses zu besorgen. Dementsprechend wurde Den Haag als Tagungsort gewählt. Die Föderalisten wurden eingeladen, sich als Mitveranstalter an dem Kongress zu beteiligen und zu diesem Zweck auch das Verbindungskomitee auszubauen. ${ }^{14}$

Für die Föderalisten stellte diese Einladung ein Danaergeschenk dar: Nahmen sie sie an und gingen ein Bündnis mit den konservativen Spitzenpolitikern und Wirtschaftsführern ein, so drohte ,die schöpferische und revolutionäre Dynamik verloren zu gehen, die die föderalistische Doktrin mit sich gebracht hatte“ und deren Durchbruch sie sich von den „Generalständen“ erhofften. Beharrten sie dagegen auf ihren eigenen Kongress-Plänen, so spalteten

11 Vgl. Lipgens: Anfänge, 1977, S. 620-623.

12 Ebenda, S. 612-617.

13 Aufzeichnung Hendrik Brugmans 24.9.1947, in: Lipgens/Loth: Documents IV, 1990, S. 41-42.

14 Protokoll eines Treffens Sandys, van Zeeland, Retinger, Kerstens 28.9.1947 in Brüssel, zitiert bei Lipgens: Anfänge, 1977, S. 627. 
sie nicht nur die europäische Bewegung, sondern gingen auch „das Risiko rascher Destruktion und Niedergang zu einer Sekte ein". ${ }^{15}$ Den Ausschlag gab schließlich der höhere Realitätsgehalt des britischen Projekts: Es würde auf jeden Fall durchgeführt werden und beträchtliche Resonanz haben; demgegenüber war unklar, wie die „Generalstände“ finanziert werden könnten und ob sie angesichts der Konkurrenzveranstaltung der etablierten Kräfte noch die angestrebte Wirkung haben konnten. Insbesondere Brugmans warb in diesem Sinne für die Annahme der Einladung. Marc und auch die italienischen Föderalisten um Altiero Spinelli waren im Grunde dagegen, hielten sich aber zurück. Am 15. November 1947 beschloss das Zentralkomitee der UEF, sich am Haager Kongress zu beteiligen und dem Ausbau des Verbindungskomitees zu einem „Koordinierungskomitee“ zuzustimmen. ${ }^{16}$

In der vagen Hoffnung, den Haager Kongress vielleicht doch noch ,,in Generalstände Europas verwandeln“ zu können, ${ }^{17}$ nahmen die Föderalisten es hin, im Koordinierungskomitee mit einem Viertel der Stimmen in der Minderheit zu sein; UEM, französischer Rat und ILEC, die programmatisch auf einer Linie lagen, verfügten jeweils über die gleiche Stimmenzahl. Den Vorsitz mussten sie Sandys überlassen, den Posten eines Sekretärs Retinger. ${ }^{18}$ Notgedrungen akzeptierten sie auch die Vorgaben für die Organisation des Kongresses, die Sandys auf einer weiteren Zusammenkunft des Komitees am 13. und 14. Dezember 1947 präsentierte: Er sollte ,in eindrucksvoller Weise die mächtige und weitreichende Unterstützung der europäischen Idee demonstrieren, die bereits existiert“ und „Material zur Diskussion, Propaganda und technischen Studien produzieren“. Dazu sollte er so repräsentativ wie möglich zusammengesetzt sein; die Entscheidung über die Einladungen sollte aber dem Koordinationskomitee vorbehalten bleiben. Als Name für die Veranstaltung wurde ,Congress of Europe " festgelegt, das Präsidium des Kongresses wurde Churchill angetragen. ${ }^{19}$

In der Praxis bedeutete die Entscheidung über die Einladungspolitik, dass Sandys und Retinger Vorschläge über einzuladende Persönlichkeiten sammelten, danach entschieden, an wen Einladungen ausgesprochen wurden, und schließlich die Zusagen registrierten. Was die Zahl der Delegierten pro Land betraf, so setzte Sandys eine Formel gemäßigter Repräsentativität durch: 15 Repräsentanten pro Land plus zwei weitere für je eine Million Einwohner. Für Frankreich ergab das 104 Delegierte, für Großbritannien 118, für Belgien und die Niederlande jeweils 33, und so weiter. Länder, deren Regierungen Vertretern des Koordinierungskomitees die Einreise verweigerten und keine Zusagen gaben, Bürger ihres Landes mit den nötigen Visa für die Teilnahme am Kongress auszustatten, sollten nur mit kleinen Beobachter-Gruppen vertreten sein. ${ }^{20}$ Das lief darauf hinaus, das westliche Europa so umfassend wie möglich zu sammeln und im Übrigen den Selbstausschluss der Sowjetunion und der von ihr beherrschten Länder noch einmal zu bekräftigen.

Zur organisatorischen Abwicklung ließ sich das Koordinierungskomitee von einer großen niederländischen Bank ein repräsentatives Büro einrichten. Kerstens sammelte so viele Spendengelder ein, dass den über 700 Teilnehmern des Kongresses nicht nur ein kostenloser

15 So die nachträgliche Charakterisierung des Dilemmas durch Denis de Rougement: The Campaign of the European Congresses, in: Government and Opposition 3/1967, S. 329-349, hier S. 338.

16 Lipgens: Anfänge, 1977, S. 630-631.

17 So Alexandre Marc in einem Schreiben an Marceau Pivert 10.12.1947, zitiert bei Lipgens: Anfänge, 1977, S. 632.

18 Ebenda, S. 628-629; Text des Agreements vom 11.11.1947, in: Lipgens/Loth: Documents IV, 1990, S. 325328.

19 Entwurf Sandys 11.12.1947, in: Lipgens/Loth: Documents IV, 1990, S. 328-339; Auszüge aus dem Sitzungsprotokoll bei Lipgens: Anfänge, 1977, S. 636-637.

20 Anhang A zum Entwurf Sandys 11.12.1947. Als „Anhang B“ legte Sandys gleich eine exemplarisch gemeinte Liste von möglichen Mitgliedern der britischen Delegation bei. 
Aufenthalt in Den Haag, sondern auch die Übernahme aller Reisekosten angeboten werden konnte. Angesichts der immer noch prekären Verhältnisse im kriegszerstörten Europa war das eine bemerkenswerte Leistung, die für den Erfolg des Unternehmens ganz entscheidend war. Als sich dennoch eine Lücke in der Finanzierung des Kongresses auftat, ließ sich Sandys von Prinz Bernhard der Niederlande beim Vorstand des Philips-Konzerns einführen. Dieser half dann mit einer sehr großzügigen Spende aus. Ende Januar 1948 wurde der Termin des Kongresses definitiv auf den 7. bis 10. Mai 1948 festgelegt; danach konnte Retinger als Sekretär die offiziellen Einladungen verschicken. ${ }^{21}$

Sandys, Retinger und auch Brugmans suchten prominente Persönlichkeiten in den verschiedenen Ländern auf, um sie zur Mitarbeit an dem Unternehmen zu bewegen. In den meisten Fällen waren sie damit erfolgreich. „Wir haben eine sehr große Arbeit geleistet“, konnte Retinger dem ehemaligen rumänischen Außenminister Gregor Gafencu schon zum Jahresende 1947 berichten. „Von den großen Staatsmännern (aber das ist noch vertraulich) haben uns ihre Unterstützung zugesagt: Mister Churchill und Sir Stafford Cripps aus Großbritannien, die Monsieurs Herriot und L. Blum aus Frankreich, die Herren van Zeeland und Spaak aus Belgien, sowie Sforza aus Italien. Die niederländische Regierung mit ihrem Ministerpräsidenten an der Spitze wird uns dort empfangen, wo unsere Sitzungen stattfinden werden: im historischen Rittersaal."22 Paul Ramadier und der italienische Ministerpräsident Alcide de Gasperi sagten ihre Teilnahme ebenfalls zu. In den westdeutschen Besatzungszonen konnten die Organisatoren unter anderen den Ministerpräsidenten von Nordrhein-Westfalen Karl Arnold und die Regierenden Bürgermeister von Hamburg und Bremen, Max Brauer und Wilhelm Kaisen gewinnen, ebenso Konrad Adenauer als Vorsitzenden der CDU in der britischen Zone, Martin Niemöller vom Rat der Evangelischen Kirche Deutschland, Gustav Heinemann als Justizminister von Nordrhein-Westfalen, Thomas Dehler, Heinrich von Brentano und Walter Hallstein als Vorsitzenden der Süddeutschen Rektorenkonferenz. ${ }^{23}$

Ebenso gelang es, die christdemokratischen ,Nouvelles Equipes Internationales“ (NEI) für die Mitarbeit an dem Kongressvorhaben zu gewinnen. Vom Februar 1948 an gehörten sie als weitere einladende Organisation dem Koordinierungskomitee an. ${ }^{24}$ Dagegen blieben alle Bemühungen der Föderalisten vergeblich, auch das sozialistische ,Comité international pour les Etats-Unis socialistes d'Europe' (EUSE) mit ins Boot zu nehmen. Seine britischen Mitglieder ,,verfielen auf jede einfache Nennung des Namens Churchill in einen Trance-Zustand und jede Möglichkeit vernünftigen Verstehens lag in Ohnmacht", klagte Henri Frenay von der französischen Sektion des Komitees nach einem Besuch in London. Mit neun zu sieben Stimmen lehnte der Vorstand des Komitees eine Beteiligung am Haager Kongress ab. ${ }^{25}$ Coudenhove-Kalergi weigerte sich, die Vereinbarung über die Bildung des Verbindungsko-

21 Niess: Europäische Idee, 2001, S. 181-182.

22 Retinger an Gafencu 27.12.1947, zitiert bei Niess: Europäische Idee, 2001, S. 173.

23 Vgl. Christoph Stillemunkes: The Discussion on European Union in the German Occupation Zones, in: Lipgens/Loth: Documents III, 1988, S. 441-565, hier S. 454.

24 Lipgens: Anfänge, 1977, S. 633; vgl. Heribert Gisch: The ,Nouvelles Équipes Internationales“ (NEI), in: Lipgens/Loth: Documents IV, 1990, S. 477-540; Michael Gehler/Wolfram Kaiser (Hrsg.): Transnationale Parteienkooperation der europäischen Christdemokraten. Dokumente 1945-1965, München 2004; Wolfram Kaiser: Christian Democracy and the Origins of European Union, Cambridge/New York 2007, S. 191-205.

25 Henry Frenay an Marcel Hytte 15.12.1947, zitiert bei Lipgens: Anfänge, 1977, S. 633; vgl. Wilfried Loth: Sozialismus und Internationalismus. Die französischen Sozialisten und die Nachkriegsordnung Europas 19401950, Stuttgart 1977, S. 199-201; Wilfried Loth: The Mouvement pour les États-Unis d'Europe (MSEUE), in: Lipgens/Loth: Documents IV, 1990, S. 277-318. 
mitees vom Juli 1947 zu ratifizieren und nannte dann immer neue Bedingungen für seine Mitarbeit, die im Grunde darauf hinausliefen, dass er selbst die Führung des Unternehmens übernahm und seine programmatische Ausrichtung kontrollierte. Erst Anfang April 1948 fand er sich zu einer Teilnahme an dem Kongress ohne Vorbedingungen bereit, zu einem Zeitpunkt, zu dem die meisten inhaltlichen Entscheidungen schon gefallen waren. Entsprechend marginal blieb sein Beitrag zur Ausrichtung des Kongresses. ${ }^{26}$

Schwerwiegender als die Absage des Sozialistenkomitees und das lange Zögern Coudenhoves und seiner Parlamentarier-Union war die Opposition des Exekutivkomitees der britischen Labour-Party. Labour-Führer wie Morgan Phillips, Hugh Dalton und Denis Healey waren entschiedene Gegner einer britischen Beteiligung an einem supranationalen Europa. In dem Kongress-Plan sahen sie darum ein höchst gefährliches Unternehmen, das zudem die Handlungsfreiheit von Außenminister Ernest Bevin beeinträchtigte und der konservativen Opposition Auftrieb gab. Auf einer Konferenz der sozialistischen Parteien aller Länder, die sich am Marshall-Plan beteiligten, am 21. und 22. März 1948 in London setzten sie die Entscheidung durch, der Einladung des Koordinierungskomitees nicht zu folgen; 40 LabourAbgeordnete, die sich schon zur Teilnahme am Haager Kongress entschlossen hatten, wurden aufgefordert, ihre Zusage rückgängig zu machen. Um die Solidarität des internationalen Sozialismus zu wahren, verboten die Parteivorstände der französischen SFIO und der SPD ihren Funktionsträgern ebenfalls die Teilnahme. ${ }^{27}$

Nicht alle sozialistischen oder sozialdemokratischen Europapolitiker ließen sich von den Verboten beeindrucken. So hielten 23 der 40 Labour-Abgeordneten an ihrer Zusage fest, darunter Ronald W. G. Mackay, der Initiator der ,All-Party Group for European Unity “ im britischen Unterhaus, der unterdessen als Stellvertreter Coudenhoves auch eine führende Rolle in der Europäischen Parlamentarier-Union spielte. León Blum, Paul Henri Spaak, Carlo Schmid und Max Brauer blieben dem Kongress fern; dagegen nahmen Paul Ramadier und Wilhelm Kaisen in offener Auflehnung gegen die Beschlüsse ihrer Parteivorstände teil. Sie konnten freilich nicht verhindern, dass die Veranstaltung eine liberal-konservative Schlagseite bekam. Ganz so repräsentativ, wie Sandys es mit guten Gründen angestrebt hatte, ${ }^{28}$ wurde der Haager Kongress nicht.

\section{Verhandlungen und Beschlüsse}

Dennoch hatte die Versammlung, die am Nachmittag des 7. Mai 1948 in Anwesenheit von Prinzessin Juliane und Prinz Bernhard der Niederlande eröffnet wurde, starkes politisches Gewicht. Insgesamt 722 Delegierte waren der Einladung schließlich gefolgt; dazu kamen etwa 250 Gäste und journalistische Beobachter. Die stärkste Delegation stellte mit 185 Mitgliedern Frankreich; ihr gehörten neben Ramadier unter anderen Edouard Bonnefous, Edouard Daladier, Edgar Faure, André François-Poncet, Edmond Giscard d'Estaing, Pierre-Olivier Lapie, François de Menthon, François Mitterrand und Paul Reynaud an. Aus Großbritannien kamen 147 Delegierte, darunter Anthony Eden und Harold Macmillan. Italien war nicht ganz so prominent vertreten: De Gasperi und andere führende Politiker mussten wegen der Regierungsneubildung im Anschluss an die Wahlen vom 18./19. April

26 Lipgens: Anfänge, 1977, S. 633-635; Martin Posselt: Richard Coudenhove-Kalergi und die Europäische Parlamentarier-Union, Diss. Graz 1987, S. 234-237, 275-284; Heribert Gisch: The European Parliamentary Union (EPU), in: Lipgens/Loth: Documents IV, 1990, S. 112-185.

27 Loth: Sozialismus, 1977, S. 204-209; Clemens A. Wurm: Great Britain: Political Parties and Pressure Groups in the Discussion on European Union, in: Lipgens/Loth: Documents III, 1988, S. 628-762; Wilfried Loth: The Socialist International, in: Lipgens/Loth: Documents IV, 1990, S. 436-475.

28 Vgl. seine Argumentation im Strategiepapier vom 11.12.1947, in: Lipgens/Loth: Documents IV, 1990, S. 330. 
absagen. Die Vertreter Portugals, für die immerhin 20 Plätze vorgesehen waren, blieben nach einer Missfallenskundgebung Salazars ganz zu Hause. Polen, Ungarn, die Tschechoslowakei, Bulgarien und Rumänien waren nur durch Exilpolitiker vertreten. Spanien musste sich mit einer Beobachter-Rolle begnügen; sie wurde von vier Delegierten wahrgenommen, an ihrer Spitze der Philosoph und Ex-Minister Salvador de Madariaga. Jeweils nur ein Delegierter kamen aus Island und der Türkei. ${ }^{29}$

Die Deutschen waren der politischen Zielsetzung entsprechend, wie sie Churchill vorgegeben hatte, als Delegierte mit vollem Status geladen. Da es darüber im Koordinierungskomitee noch einmal zu Diskussionen gekommen war, hatte sich ihre Einladung verzögert. Für manche der Eingeladenen konnten daraufhin nicht mehr rechtzeitig die erforderlichen Ausreisegenehmigungen der Besatzungsbehörden und Devisen beschafft werden, sodass die deutsche Delegation schließlich nur 51 Persönlichkeiten zählte. Sie genossen es sehr, von Churchill in seiner Eröffnungsansprache ausdrücklich als notwendige Partner beim Aufbau Europas begrüßt zu werden und zum ersten Mal seit Kriegsende auf internationaler Ebene wieder auf gleicher Augenhöhe auftreten zu können. In den Diskussionen des Kongresses hielten sie sich allerdings meist zurück, im Bewusstsein fortdauernder Abhängigkeit von den Entscheidungen der Besatzungsmächte. Stattdessen nutzen sie die Gelegenheit, Kontakte mit den prospektiven Partnern zu knüpfen. So traf Adenauer nach der Eröffnungsveranstaltung zum ersten Mal mit Churchill zusammen und fand sich ,von ihm mit gewinnender Freundlichkeit behandelt" . ${ }^{30}$ Innerhalb der deutschen Delegation lernte er Walter Hallstein kennen, der später sein engster europapolitischer Mitarbeiter werden sollte. ${ }^{31}$

Auf Churchills Eröffnungsansprache folgten Reden von Ramadier, Coudenhove-Kalergi, Brugmans $^{32}$ und van Zeeland. Ein Versuch der Föderalisten, die Veranstaltung durch Verlesen einer „Präambel“, die auf die Schaffung einer europäischen Versammlung der ,lebendigen Kräfte aller unserer Nationen" zielte, gleich nach der Rede Churchills doch noch stärker in die Richtung von „Generalständen“ zu ziehen, wurde abgewiesen. Denis de Rougement, der die Proklamation einer solchen Zielsetzung des Kongresses zur Bedingung für seine Mitarbeit an der Kongressvorbereitung gemacht hatte, konnte den von ihm redigierten Text nur als „Botschaft an die Europäer“ verlesen, nach Statements von Sandys, de Madariaga und Ramadier. Die den Föderalisten zunächst zugestandene Unterzeichnung der Botschaft durch alle Delegierten unterblieb, nachdem einige Teilnehmer Einspruch gegen die Forderung nach einer gemeinsamen Verteidigung erhoben hatten. ${ }^{33}$

Auf der anderen Seite sorgte die Kongressregie aber auch dafür, dass Churchill die Veranstaltung keineswegs dominieren konnte. Die Mitglieder des Koordinierungskomitees waren schon fünf Tage vor Kongressbeginn angereist und hatten sich im Detail über den Ablauf der Veranstaltung verständigt. ${ }^{34}$ Die Berichte $\mathrm{zu}$ politischen, wirtschaftlichen und kulturellen Fragen, an denen die Komiteemitglieder seit Jahresbeginn in unterschiedlicher Zusammensetzung gearbeitet hatten, wurden nicht einfach zur Abstimmung gestellt, sondern in entsprechenden Ausschüssen des Kongresses während des ganzen zweiten Verhand-

29 Niess: Europäische Idee, 2001, S. 183-184 und 190-192.

30 Konrad Adenauer: Erinnerungen 1945-1953, Stuttgart 1965, S. 210.

31 Walter Hallstein: United Europe. Challenge and Opportunity, Cambridge, Mass. 1962, S. 8. Weitere Zeugnisse zur deutschen Delegation bei Niess: Europäische Idee, 2001, S. 193-197.

32 Text in Lipgens/Loth: Documents IV, 1990, S. 51-55.

33 De Rougement: Campaign, 1967, S. 339-345; Reden und Resolutionen in: Europe Unites. The story of the campaign for European Unity, including a full report of the Congress of Europe, held at The Hague, London 1949; Verbatim report des Kongresses (hektographiert) im Archiv der Europäischen Bewegung, Deposit in den Historischen Archiven der Europäischen Union, Fonds ME, Florenz.

34 De Rougement: Campaign, 1967, S. 342. 
lungstages und dann noch einmal am Abend des dritten Tages intensiv diskutiert und dabei zum Teil auch noch einmal substanziell verändert. Eugen Kogon, der von der UEF als deutscher Teilnehmer rekrutiert worden war, hielt gleich nach der Rückkehr aus Den Haag in seinen ,Frankfurter Heften“ fest: „Den beteiligten Sozialisten, Christlich-Sozialen, Syndikalisten und Fortschrittlichen gelang es eindeutig zu verhindern, dass Churchill, dessen Bedeutung für die Einigung Europas im übrigen von jedermann anerkannt wird, und seine meist ebenso reichen wie stockkonservativen Gefolgsleute dem Kongress ihr Gepräge geben konnten." 35

Die Verhandlungen des Politischen Ausschusses wurden von Auseinandersetzungen um das Zielbild, die Methoden und das Tempo der europäischen Einigung geprägt. Indirekt stand damit auch die Frage einer britischen Beteiligung im Raum, obwohl das vielen Teilnehmern gar nicht bewusst war. Sandys hatte in einem Rahmenentwurf für die Politische Resolution des Kongresses, den er Ende 1947 unter den Komiteemitgliedern zirkulieren ließ, verlangt, der Kongress solle sich „für das Endziel der europäischen Einheit“ aussprechen, dabei aber nur , in höchst allgemeinen Formulierungen die verschiedenen Formen erläutern, die diese Einheit annehmen könnte“. Als Institution zur Förderung des Einigungsprozesses wollte er einen „Europäischen Rat“ fordern, bestehend aus „einem System regelmäßiger Konferenzen europäischer Minister“, die ,soweit als möglich einen gemeinsamen europäischen Standpunkt entwickeln sollten“, und einem „ständigen internationalen Sekretariat", das die laufenden europäischen Probleme studiert und dem Rat Vorschläge unterbreitet. ${ }^{36}$

In dem Bericht, der den Delegierten nach der Abstimmung im Koordinierungskomitee vorgelegt wurde, wurde auf Drängen der Föderalisten die supranationale Dimension der zu schaffenden „Politischen Union“ klarer angesprochen: „Früher oder später“ müsse der schrittweise politische Zusammenschluss ,den Verzicht auf, oder um genauer zu sein, die gemeinsame Ausübung gewisser Souveränitätsrechte einschließen“. Als Endziel der Entwicklung wurde ,die Bildung einer vollständigen Föderation mit einem gewählten europäischen Parlament“ genannt. Der Europäische Rat wurde jetzt als „Emergency Council“ bezeichnet, der ,für die Durchführung gemeinsamer Aktionen zur Sicherung nicht nur des wirtschaftlichen Wiederaufbaus und der militärischen Verteidigung, sondern auch des Erhalts der demokratischen Freiheit verantwortlich“ sein sollte. Außerdem sollte der Rat „die weiteren Stufen der politischen und wirtschaftlichen Integration Europas planen“. Hinzu kam die Forderung nach Schaffung einer „Europäischen Beratenden Versammlung“, die den Europäischen Rat „unterstützen und beraten“ sollte. Ihre Mitglieder sollten zunächst von den nationalen Parlamenten entsandt werden; ,,später“ sollte „ein System direkter Wahl eingerichtet werden". 37

In den Verhandlungen des Ausschusses rückte unter dem Einfluss von Mackay die Idee einer Europäischen Versammlung in den Mittelpunkt des Forderungskatalogs. Sie sollte „sofortige praktische Maßnahmen empfehlen, die geeignet sind, die notwendige wirtschaftliche und politische Union Europas in fortschreitendem Maße zu verwirklichen“ und Pläne für ,die rechtlichen und verfassungsmäßigen Folgerungen“ ausarbeiten, „die sich aus der Schaffung einer derartigen Union oder Föderation ergeben“. Die Forderung nach Einrichtung eines Europäischen Rates entfiel. Stattdessen wurde erklärt, ,dass die Zeit gekommen“

35 Eugen Kogon: Der Haager Europäische Kongreß, in: Frankfurter Hefte 6/1948, S. 481-483, hier S. 481-482.

36 Duncan Sandys: Suggested Outline of the Political Report for the Hague Congress, 21.12.1947, in: Lipgens/ Loth: Documents IV, 1990, S. 330-332.

37 Lipgens/Loth: Documents IV, 1990, S. 333-338. 
sei, ,zu der die europäischen Nationen einen Teil ihrer Souveränitätsrechte übertragen und verschmelzen müssen“".38 Die Forderung der italienischen Föderalisten, aus der Europäischen Versammlung gleich eine Verfassunggebende Versammlung zu machen, wurde mit großer Mehrheit abgelehnt. Auch für einen Antrag Reynauds, die Direktwahl der Versammlung schon für die erste Wahlperiode zu verlangen, konnten sich nur wenige Delegierte begeistern. Zu Recht warnte Mackay davor, die britische und auch die französische Regierung mit einem solchen Vorschlag zu provozieren: Er würde die Chancen nur mindern, tatsächlich eine Versammlung zu bekommen, die mit der Ausarbeitung eines mehrheitsfähigen Verfassungsentwurfs beauftragt wurde. Die Resolution wurde schließlich in der Nacht zum 10. Mai verabschiedet. Von den über 300 Delegierten, die im Politischen Ausschuss mitgearbeitet hatten, stimmten weniger als ein Dutzend dagegen. ${ }^{39}$

Im Wirtschafts- und Sozialausschuss stießen die liberalen Integrationsvorstellungen der ILEC, die bei der Vorbereitung federführend gewesen war, ${ }^{40}$ auf die Kritik der Sozialisten und Gewerkschaftsvertreter. Das Zielbild der anzustrebenden „wirtschaftlichen Union“ blieb damit notwendigerweise etwas vage. Immerhin wurde aber festgehalten, dass der europäische Wiederaufbau „,nicht erfolgreich sein“ könne, ,,wenn er auf der Grundlage streng geteilter nationaler Staatshoheiten durchgeführt wird“ und ,,auf jeder Stufe von einer gleichlaufenden Politik immer engeren politischen Zusammenschlusses begleitet wird“. Als Maßnahmen wurden gefordert: die schrittweise Beseitigung der Handels- und Zollschranken, ein gemeinsamer, wenn auch niedriger Außenzoll, Haushaltsstabilisierung, Angleichung von Preisen und Löhnen, freie Konvertierbarkeit der Währungen und schließlich eine Währungsunion, ebenso gemeinsame Planung zur Entwicklung der Landwirtschaft und der Grundindustrien und eine „Gleichschaltung“ der Haushalts- und Kreditpolitik sowie der Sozialgesetzgebung. ${ }^{41}$

Der Kritik der Linken kam die Mehrheit der Kommission mit Bekenntnissen zu sozialstaatlichen Prinzipien entgegen. So wurden die beruflichen, wirtschaftlichen und sozialen Organisationen der einzelnen Länder aufgefordert, ,,Mittel und Wege zu einer weiteren Steigerung der Erzeugung und Vereinfachung der Verteilung bei gleichzeitiger Besserung der sozialen Verhältnisse und Sicherung einer gerechten Verteilung der Erzeugnisse der wirtschaftlichen Tätigkeit gemeinsam zu untersuchen“. Die Förderung der Freizügigkeit der Arbeitskräfte sollte mit „Sicherung des Lohnstandards und der sozialen Sicherheit“ verbunden werden; die Wirtschaftspolitiken der einzelnen Länder sollten einander ,,angeglichen“ werden, ,um eine Vollbeschäftigung zu gewährleisten““42 Auf konkrete Maßnahmen wie eine supranationale Kontrolle des Kapitalverkehrs und eine Europäisierung der Ruhrindustrie wollte sich die Mehrheit der Delegierten jedoch nicht festlegen. Auch für eine Unterstützung der Forderung nach einer Beteiligung der Arbeiter und ihrer Organisationen an den europäischen Gremien fand sich keine Mehrheit. Der Protest der Gewerkschaftsvertreter gegen diese Abfuhr konnte nur dadurch aufgefangen werden, dass der Ausschuss nach langwierigen Verhandlungen - die bis in den frühen Morgen des 10. Mai andauerten - einem ,,post-

38 Politische Resolution des Haager Kongresses, in: Lipgens/Loth: Documents IV, 1990, S. 345-347; deutsche Übersetzung, in: Walter Lipgens (Hrsg.): 45 Jahre Ringen um die Europäische Verfassung. Dokumente 19391984, Bonn 1986, S. 240-242. Bei der häufig nachgedruckten Übersetzung im Europa-Archiv 3 (1948), S. 1443-1444, handelt es sich um den vorletzten Entwurf, nicht um die verabschiedete Fassung.

39 Auszüge aus den Debattenbeiträgen, in: Lipgens/Loth: Documents IV, 1990, S. 339-345. Vgl. auch Henri Brugmans: L'idée européenne 1920-1970, Bruges 1970, S. 133.

40 Text des vorgelegten Berichts, in: Lipgens/Loth: Documents IV, 1990, S. 208-212.

41 Wirtschaftliche und soziale Resolution, in: Lipgens/Loth: Documents IV, 1990, S. 347-350; deutsche Übersetzung, in: Europa-Archiv 3 (1948), S. 1444-1445.

42 Ebenda. 
Congress Economic Committee“ den Auftrag gab, „eine Kompromiss-Politik für Europa auszuarbeiten, die die besten Grundzüge von Kapitalismus und Sozialismus enthalten sollte". ${ }^{43}$

Dem Kulturellen Ausschuss wurde ein Bericht vorgelegt, den Denis de Rougement im Kontakt mit Autoren wie Étienne Gilson, Ignazio Silone und Salvador de Madariaga erarbeitet hatte. Er sprach von einem ,gemeinsamen christlichen Erbe sowie anderen geistigen und kulturellen Werten“ und der ,gemeinsamen Verpflichtung gegenüber den menschlichen Grundrechten“, die für die Mitglieder einer ,europäischen Union“ gelten sollten. Allzu föderalistische Passagen waren nach einer Intervention Retingers im letzten Moment aus der Vorlage entfernt worden. Konkret wurde dann die Einrichtung eines ,europäischen Kulturzentrums“ verlangt, das unabhängig von jeder Regierungsüberwachung „das Bewusstsein der europäischen Einheit fördern“ und als Forum des Austauschs der ,geistigen Führer“ Europas dienen sollte. Weiter wurde die Schaffung einer ,europäischen Zentrale für Kinder und Jugendliche“ gefordert, die Kinder- und Jugendforschung betreiben sowie „den Austausch von Jugendlichen aller Schichten in Europa“ fördern sollte. Zuletzt sah der Entwurf auch noch die Schaffung eines Obersten Gerichtshofs zur Einhaltung der Menschenrechte vor, der sowohl von einzelnen Bürgern als auch von ,kollektiven Einheiten“ angerufen werden konnte und „,befähigt“ sein sollte, ,die Einhaltung der Erklärung der Menschenrechte zu sichern". 44

Die Debatte über den kulturellen Bericht „entfaltete sich in der üblichen Konfusion“, wie de Rougement sarkastisch kommentierte. Während der Schriftsteller Charles Morgan die kulturellen Angelegenheiten ganz in den Händen der nationalen Regierungen lassen wollte, verlangten andere Delegierte die sofortige Einrichtung einer Körperschaft, die die Arbeit des Kongresses fortsetzen sollte. Ein Hauptmann Cheshire von der Bewegung für moralische Aufrüstung wollte die Rückkehr zu Gott in dem Dokument verankert sehen und griff den Entwurf als ,,anti-christlich“ an. Schließlich sprach sich Bertrand Russell nachdrücklich für das vorgeschlagene Kulturzentrum aus: Es werde den Menschen der verschiedenen Länder helfen, engen Kontakt zu halten und die Standpunkte der jeweils anderen verstehen zu lernen. Seine Autorität trug dazu bei, dass die materiellen Vorschläge des Berichts schließlich alle einstimmig angenommen wurden..$^{45}$

\section{Meilenstein in der Entwicklung zur Einheit}

Einige dezidierte Föderalisten waren vom Verlauf des Kongresses so enttäuscht, dass sie kurz vor seinem Ende unter Protest abreisen wollten. Sandys musste van Zeeland als Vermittler einschalten, um einen solchen offenen Bruch zu vermeiden. Möglicherweise war die Verlesung der „Botschaft an die Europäer“ der Preis, den er dafür zahlen musste - er selbst hatte sie nach den Einwänden gegen ein Bekenntnis zur europäischen Verteidigung zunächst ganz streichen wollen. ${ }^{46}$ Marc setzte dann aber eine Presseerklärung der UEF durch, in der

43 Europe Unites, S. 48. Vgl. die Diskussionsbeiträge ebenda, S. 46-65 sowie die Darstellung bei Loth: Sozialismus, 1977, S. 210.

44 Resolution des Kulturausschusses, in: Europa-Archiv 3 (1948), S. 1445-1446; zur Entstehung de Rougement: Campaign, 1967, S. 339-341. Leider gibt de Rougement nicht an, worin genau die Korrekturen bestanden, die er nach einer Besprechung am 26. April 1948 in London vornehmen musste.

45 Ebenda, S. 342

46 De Rougement ist auch hier nicht ganz präzise: Er berichtet von einer Krisensitzung, in der van Zeeland die Verlesung der Botschaft ohne die inkriminierte Passage als Kompromiss vorgeschlagen hat (ebenda, S. 344), erwähnt aber die Abreisedrohung nicht. Über diese informierte Sandys Frank Niess in einem Gespräch am 25.11.1966; siehe Niess: Europäische Idee, 2001, S. 210. 
ungenügende Repräsentativität des Kongresses und „Halbheiten“ in seinen Beschlüssen kritisiert wurden. ${ }^{47}$ Auf dem zweiten Jahreskongress der UEF vom 7. bis 11. November 1948 in Rom musste Brugmans für seine Bereitschaft zur Zusammenarbeit mit dem UEM heftige Kritik einstecken. ${ }^{48}$

Die Enttäuschung der radikalen Föderalisten über das Ausbleiben eines Durchbruchs zur europäischen Konstituante sollte jedoch nicht den Blick dafür verstellen, dass es den Initiatoren des Haager Kongresses tatsächlich gelungen war, die europäische Einigung auf die Agenda westeuropäischer Politik zu setzen. Nachdem das Koordinierungskomitee den AuBenministern der fünf Mitgliedstaaten des Brüsseler Paktes am 18. Juli 1948 ein Memorandum übermittelt hatte, in dem der Vorschlag einer ,Europäischen Versammlung“ von Parlamentsvertretern erläutert wurde, machte sich der französische Außenminister Georges Bidault diesen Vorschlag bei der nächsten Sitzung des Konsultativrats des Brüsseler Paktes am 20. Juli 1948 in Den Haag zu eigen. Knapp einen Monat später, am 18. August 1948, billigte die unterdessen umgebildete französische Regierung einen Verfahrensvorschlag des Koordinierungskomitees zur Einberufung dieser Versammlung. Damit war entschieden, dass bei der nächsten Zusammenkunft des Konsultativrats am 25. Oktober 1948 über diesen Vorschlag befunden werden musste. ${ }^{49}$

In den Regierungsverhandlungen über das Projekt einer Europäischen Versammlung und der Auseinandersetzung über die Ergebnisse der ersten Sitzungsperiode des daraus hervorgehenden Europarats sollte sich herausstellen, dass die Labour-Regierung für eine substanzielle Beteiligung Großbritanniens an dem europäischen Einigungswerk nicht zu haben war. ${ }^{50}$ Das ändert aber nichts daran, dass der transnationale gesellschaftliche Konsens, auf dem die späteren europäischen Gemeinschaften beruhten, in erster Linie von britischen Europapolitikern organisiert worden war und dass er auf ein weit größeres Europa zielte als das „Europa der Sechs“, das dann zunächst zustande kam. Dieser Konsens war notwendigerweise unpräzise, was die institutionelle Ausgestaltung eines vereinten Europas betraf. Er umfasste aber sehr klar die Bereitschaft zur partiellen Zusammenlegung nationaler Souveränitätsrechte, zur sozialstaatlichen Ausgestaltung der Gemeinschaft und zur Festigung der demokratischen Ordnung in den beteiligten Ländern. ${ }^{51}$ Nach dem Haager Kongress ging er nicht einfach wieder verloren. Vielmehr entwickelte er sich in kritischer Auseinandersetzung mit den Erfahrungen konkreter Europapolitik, und diese wurde von ihm mitgeprägt.

Wenige Wochen nach dem Haager Kongress gab sich Winston Churchill von seinem Erfolg überzeugt: „Dieser Europa-Kongress wird von den Historikern als Meilenstein in der Entwicklung unseres Kontinents hin zur Einheit gewürdigt werden. "52 Bislang ist das wohl noch nicht in genügendem Maße geschehen; die historische Forschung hat sich allzu sehr auf das Regierungshandeln und seine Interpretation in diplomatischen Akten konzentriert. Nachdem die Europäische Union heute aber in vielerlei Hinsicht so aussieht, wie sie von den Delegierten des Haager Kongresses in engagierten Auseinandersetzungen skizziert worden war, spricht in der Tat Vieles dafür, den Kongress von 1948 als einen Erinnerungsort des gemeinschaftlichen Europas zu entdecken.

47 Erklärung 20.5.1948, in: Lipgens/Loth: Documents IV, 1990, S. 56.

48 Ebenda, S. 57-78.

49 Vgl. Loth: Der Weg nach Europa, 1990, S. 70-72; das Memorandum vom 18.7.1948, in: Lipgens/Loth: Documents IV, 1990, S. 359-360, der Verfahrensvorschlag vom 18.8.1948 ebenda, S. 360-362.

50 Loth: Weg nach Europa, 1990, S. 70-79.

51 Die auf die radikalen Föderalisten zurückgehende Charakterisierung Sandys " und der Komiteemehrheit als „Unionisten“ ist irreführend.

52 Festgehalten von Sandys in einem Memorandum für das Koordinierungskomitee, zitiert bei Niess: Europäische Idee, 2001, S. 219. 